Article

\title{
Isolated Neutropenia/Benign Ethnic Neutropenia: A Common Clinical and Laboratory Finding in Southern and Southwestern Saudi Arabia
}

\author{
Zuhier A. Awan 1,2, Saeed M. Al Amoudi ${ }^{2}$, Muhammad Saboor ${ }^{3,4}$ and Husain Alkhaldy 5,6* \\ 1 Department of Clinical Biochemistry, Faculty of Medicine, King Abdulaziz University, Saudi Arabia; \\ z.awan@alborglaboratories.com \\ 2 Department of Clinical Pathology, Al-Borg Medical Laboratories, Saudi Arabia; z.awan@alborglaborato- \\ ries.com (ZAA), s.alamoudi@alborglaboratories.com (SMA) \\ 3 Department of Medical Laboratory Technology, Faculty of Applied Medical Sciences, Jazan University, Saudi Arabia; \\ msaboor81@gmail.com \\ 4 Medical Research Centre (MRC), Jazan University, Jazan, Saudi Arabia; msaboor81@gmail.com \\ 5 Department of Internal Medicine, College of Medicine, King Khalid University, Saudi Arabia; \\ halkhaldy@kku.edu.sa \\ 6 Research Center for Advanced Materials Science, King Khalid University, Abha, Saudi Arabia, Saudi Ara- \\ bia; halkhaldy@kku.edu.sa \\ * Correspondence: halkhaldy@kku.edu.sa; Tel.: +96672417578; Fax: +96672418111
}

\begin{abstract}
Objectives: Isolated mild neutropenia is a common clinical problem. The current study aims to validate our previous findings regarding the high prevalence of isolated neutropenia in Southern and Southwestern Saudi Arabia and explore the effect of altitude or regional differences. Methods: In this retrospective cross-sectional study, laboratory results of a commercial laboratory were screened over a period of 5 years (2016-2020) in seven different cities in South and southwestern Saudi Arabia. Participants' laboratory investigations were reviewed and excluded for any abnormal complete blood count, renal profile, liver profile, lipid profile, thyroid function test, fasting blood glucose, or $\mathrm{HbA1c}$ findings. Descriptive analysis and 95th percentile range were calculated using standard statistical methods. Results: A total of 91,880 complete blood count results were included in the final analysis. isolated neutropenia is common laboratory finding, with a prevalence ranging from $11-23 \%$. The 2.5 th percentile of the neutrophil count was lower than $1.5 \times 109 / \mathrm{L}$ in all seven cities. Conclusions: Mild to moderate neutropenia is common in Southern and Southwestern Saudi Arabia. Benign ethnic neutropenia (BEN) may explain this high prevalence. Since BEN has no clinical significance, the reference range for normal neutrophil counts needs to be adjusted to reflect the effect of BEN.
\end{abstract}

Keywords: Isolated neutropenia; benign ethnic neutropenia; reference intervals; Saudi Arabia

\section{Introduction}

Neutrophils are the most abundant leukocytes in the peripheral blood. They play a pivotal role in the innate immune system and are a key regulator of the adaptive immune system $[1,2]$. The absolute neutrophil count (ANC) normally ranges from 1.8 to $7.5 \times 10^{9} / \mathrm{L}$ [3]. Marked reduction in the peripheral neutrophil count is regarded as a serious clinical problem that needs to be addressed. Peripheral neutrophils constitute only a minute fraction of the total body neutrophils that are mainly in the bone marrow. The number of neutrophils in the peripheral circulation may be affected by multiple factors, including stress, smoking, pregnancy, and drugs, that affect the measured fraction of the neutrophil count. Altitude may also be associated with lower neutrophil counts [4]. Reduced ANC is defined as a count less than $1.5 \times 10^{9} / \mathrm{L}$ and is known as neutropenia. In severe cases, 
neutropenia predisposes an individual to infections or even life-threatening consequences. Neutropenia is categorized into three subtypes based on the ANC: mild (1.0-1.5 $\left.\times 10^{9} / \mathrm{L}\right)$, moderate $\left(0.5-1.0 \times 10^{9} / \mathrm{L}\right)$, and severe $\left(<0.5 \times 10^{9} / \mathrm{L}\right)$.

Decreased production or increased destruction of neutrophils may cause neutropenia [3]. Moreover, as first reported in 1941 by Forbes et al., low neutrophil count $\left(<1.5 \times 10^{9} / \mathrm{L}\right)$ in certain ethnicities with no predisposition to infections indicates no clinical disadvantage in the affected individuals. This condition is known as benign ethnic neutropenia (BEN) $[5,6]$.

In ethnicities with reduced circulating neutrophil counts, the bone marrow cellularity, morphology, number of granulocyte colony-forming units, and maturation of myeloid cells remain normal. However, slight reduction in the number of progenitors has been observed in some cases of BEN [5]. Additionally, a slight increase in ANC has been reported in individuals with BEN after exercise [7]. Subjects with BEN show a circadian variation of the neutrophil count similar to that observed in subjects without BEN [8].

BEN is most common in Africans, Afro-Americans, Ethiopian Jews, and Yemenite Jews [6,9-11]. We previously showed that a higher prevalence of isolated neutropenia was observed in two cities (Jeddah and Abha) in Saudi Arabia [12]. We aimed to confirm our previous findings through a large sample size from seven different cities (Jeddah, Taif, Al Baha, Abha, Khamis Mushait, Najran, and Gizan) in Southern and Southwestern Saudi Arabia. We also aimed to explore any potential association of neutropenia prevalence with altitude or any regional differences.

\section{Methods}

The study protocol was approved by the ethical and research committee of King Khalid University, Abha, Saudi Arabia (ECM\#2019-68)-(HPO-06-B-001) and was conducted in accordance with the Helsinki Declaration. In this retrospective study, results of a commercial laboratory (Al-Borg Laboratories) were screened over a period of 5 years (20162020) in seven different cities in Saudi Arabia. Laboratory investigations included complete blood count $(\mathrm{CBC})$, renal profile, liver profile, lipid profile, thyroid function test, fasting blood glucose measurement, and $\mathrm{HbA1c}$ measurement. Screened participants were either customers who do lab tests for health check-ups or follow up of chronic health conditions. Data were collected from the laboratories located in Jeddah (sea level), Taif (1,898 m above sea level), Al Baha (2000 m), Abha (2,270 m), Khamis Mushait (1,700 m), Najran (1,293 m), and Gizan (sea level). Exclusion lab criteria were applied to exclude any pre-existing health conditions. Subjects selected were all having normal CBC, biochemical, kidney, liver, sugar, lipid and thyroid profiles and thus were assumed healthy and proper to estimate the neutrophil count normal range.

\section{Inclusion and Exclusion Criteria}

Male and female patients aged 13-60 years were included. To estimate the white blood cell count (WBC) reference, the following exclusion criteria were applied: any abnormalities, unless mild, in the renal profile (serum blood urea nitrogen, creatinine), liver profile (ALT, AST, total bilirubin, and direct bilirubin), lipid profile (cholesterol, HDL, LDL, and triglyceride), thyroid function test (TSH, T4), and diabetic profile (fasting blood sugar and $\mathrm{HbA} 1 \mathrm{c})$.

For CBC, individuals with hemoglobin $(\mathrm{Hb})$ concentration of $12-19 \mathrm{~g} / \mathrm{dl}$, platelet count of $140-450 \times 10^{9} / \mathrm{L}$, and WBC count of less than $11.5 \times 10^{9} / \mathrm{L}$ were included. For low WBC count, the exclusion was applied only if other blood cell types were abnormal, e.g., concurrent anemia or thrombocytopenia. Analyses were performed with both inclusion and exclusion of neutrophil counts less than $0.5 \times 10^{9} / \mathrm{L}$.

\section{Blood Collection and Laboratory Analysis}

To ensure no inter laboratory variations in the obtained results, the different branches follow organizational standard operating protocols of blood sample collection for CBC. blood samples were collected into liquid K3 EDTA tubes and processed within 2 hours of 
collection. Sysmex automated analyzers (Sysmex Corporation, Kobe, Japan) were utilized for WBC enumeration and differential count.

\section{Statistical Analysis}

The participants' data were de-identified for any personal information before importing to an Excel datasheet. After extraction, data were revised, coded, and transferred into the statistical software IBM SPSS Statistics for Windows, version 22 (IBM Corp., Armonk, N.Y., USA). Normal distribution assessment was applied for all scale parameters. Data for both sex and age were stratified into two groups: young adults (13-40 years old) and middle-aged adults (41-60 years old). Descriptive analyses using the mean and $95 \%$ confidence interval, median, range, standard deviation (SD), and percentiles $\left(2.5^{\text {th }}, 25^{\text {th }}, 75^{\text {th }}\right.$, and $97.5^{\text {th }}$ ) were utilized to assess the normal population range. Student's t-tests were used to compare continuous variables between different sex and age groups. A p-value $<0.05$ was considered statistically significant. Since plasma volume is known to be reduced with altitude, we explored any association between neutrophil count and hematocrit considering that lower hematocrit is associated with higher plasma volume and vice versa.

\section{Results}

For the present study, 91,880 CBC results were included in the final analysis. Table 1 summarizes the frequency of neutropenia in the studied population, while Table 2 depicts the statistical analysis of the mean, SEM, median, SD, range, and percentiles $\left(2.5^{\text {th }}, 25^{\text {th }}\right.$, $75^{\text {th }}$, and $\left.97.5^{\text {th }}\right)$. Comparison between groups based on sex and age group is depicted in Table 3.

Mild neutropenia was the most common type of neutropenia among the study participants of all cities. The city of Al Baha showed slightly lower frequency in comparison with others, while the highest prevalence was observed in Najran and Gizan (17\%). A high prevalence of moderate neutropenia was observed in Abha (5\%), Najran (5\%), and Gizan $(6 \%)$. Severe neutropenia was rare among all types, with only 88 cases out of 91,880 (Table 1). Pearson correlation between hematocrit and neutrophil count was not significant.

The lowest value of ANC for the $2.5^{\text {th }}$ percentile was found in Najran $\left(0.83 \times 10^{9} / \mathrm{L}\right)$, while the highest was found in Jeddah $\left(1.05 \times 10^{9} / \mathrm{L}\right)$. Additionally, the $2.5^{\text {th }}$ percentile ANC value of the study participants in the remaining cities was between the ANC values found in Jeddah and Najran $\left(0.83-1.05 \times 10^{9} / \mathrm{L}\right)$. The highest prevalence of isolated neutropenia was found in Najran (23\% with mild and moderate forms). Gizan had a prevalence of $22 \%$, making it the second most prevalent city for neutropenia, while in Taif, Jeddah, Khamis Mushait, Abha, and Al Baha, the frequency of neutropenia (combined mild and moderate) was found to be $11 \%, 15 \%, 16 \%, 17 \%$, and $18 \%$, respectively. Severe neutropenia was the rarest, with only 88 cases out of $91,880(0.096 \%)$.

Table 1. Frequency of neutropenia in the studied populations.

\begin{tabular}{|c|c|c|c|c|c|c|c|c|}
\hline \multirow{4}{*}{ Number } & & Jeddah & Taif & Al Baha & Abha & $\begin{array}{l}\text { Khamis } \\
\text { Mushait }\end{array}$ & Najran & Gizan \\
\hline & Female & 29738 & 3486 & 1867 & 4689 & 6554 & 2188 & 2885 \\
\hline & Male & 24001 & 2765 & 1359 & 3315 & 4807 & 1840 & 2386 \\
\hline & Overall & 53739 & 6251 & 3226 & 8004 & 11361 & 4028 & 5271 \\
\hline \multirow{3}{*}{$\begin{array}{l}\text { Mild neutropenia } \\
\left(1.0-1.5 \times 10^{9} / \mathrm{L}\right)\end{array}$} & Female & 3544 & 287 & 249 & 631 & 748 & 365 & 464 \\
\hline & Male & 2744 & 200 & 190 & 310 & 564 & 302 & 412 \\
\hline & Overall & $6288(12 \%)$ & $\begin{array}{l}487 \\
(8 \%)\end{array}$ & $\begin{array}{c}439 \\
(14 \%)\end{array}$ & $\begin{array}{c}941 \\
(12 \%)\end{array}$ & $1312(12 \%)$ & $\begin{array}{c}667 \\
(17 \%)\end{array}$ & 876 (17\%) \\
\hline
\end{tabular}




\begin{tabular}{|c|c|c|c|c|c|c|c|c|}
\hline \multirow{3}{*}{$\begin{array}{l}\text { Moderate neutropenia } \\
\left(0.5-1 \times 10^{9} / \mathrm{L}\right)\end{array}$} & Female & 961 & 93 & 81 & 235 & 252 & 135 & 139 \\
\hline & Male & 712 & 69 & 55 & 156 & 198 & 119 & 106 \\
\hline & Overall & $1673(3 \%)$ & $162(3 \%)$ & $136(4 \%)$ & $391(5 \%)$ & 450 (4\%) & $254(6 \%)$ & $245(5 \%)$ \\
\hline \multirow{3}{*}{$\begin{array}{c}\text { Severe } \\
\text { neutropenia } \\
\left(<0.5 \times 10^{9} / \mathrm{L}\right)\end{array}$} & Female & 15 & 7 & 7 & 8 & 5 & 3 & 3 \\
\hline & Male & 13 & 2 & 8 & 7 & 3 & 5 & 2 \\
\hline & Overall & $\begin{array}{c}28 \\
(0.05 \%) \\
\end{array}$ & $\begin{array}{c}9 \\
(0.14 \%) \\
\end{array}$ & $\begin{array}{c}15 \\
(0.46 \%) \\
\end{array}$ & $\begin{array}{c}15 \\
(0.19 \%) \\
\end{array}$ & $\begin{array}{c}8 \\
(0.07 \%) \\
\end{array}$ & $\begin{array}{c}8 \\
(0.20 \%) \\
\end{array}$ & $\begin{array}{c}5 \\
(0.10 \%) \\
\end{array}$ \\
\hline
\end{tabular}

Table 2. Descriptive analysis of distribution of neutrophils count $\left(\times 10^{9} / \mathrm{L}\right)$ in the studied populations.

\begin{tabular}{cccccccccccc}
\hline City & $\begin{array}{c}\text { Num- } \\
\text { ber }\end{array}$ & Mean & SEM & $\begin{array}{c}\text { Me- } \\
\text { dian }\end{array}$ & SD & Min & Max & $\mathbf{2 . 5}^{\text {th }}$ per & $\mathbf{2 5}^{\text {th }}$ per & $\mathbf{7 5}^{\text {th }}$ per & $\mathbf{9 7 . 5}^{\text {th }}$ per \\
\hline Jeddah & 53741 & 3.18 & 0.01 & 3.05 & 1.35 & 0.50 & 8.98 & 1.05 & 2.14 & 4.03 & 6.19 \\
Taif & 6251 & 3.15 & 0.02 & 3.02 & 1.35 & 0.50 & 8.55 & 1.00 & 2.11 & 4.01 & 6.15 \\
Al Baha & 3226 & 2.74 & 0.02 & 2.55 & 1.29 & 0.50 & 8.65 & 0.90 & 1.73 & 3.55 & 5.61 \\
Abha & 8004 & 2.76 & 0.01 & 2.55 & 1.32 & 0.50 & 8.77 & 0.87 & 1.73 & 3.58 & 5.87 \\
Khamis Mushait & 11361 & 2.90 & 0.01 & 2.74 & 1.34 & 0.51 & 8.83 & 0.91 & 1.84 & 3.74 & 5.93 \\
Najran & 4030 & 2.56 & 0.02 & 2.40 & 1.22 & 0.52 & 7.99 & 0.83 & 1.57 & 3.34 & 5.39 \\
Gizan & 5271 & 2.43 & 0.02 & 2.16 & 1.16 & 0.50 & 8.47 & 0.89 & 1.59 & 3.05 & 5.42 \\
\hline
\end{tabular}

${ }^{1}$ Total numbers indicate all subjects analyzed after applying the exclusion criteria. Neutrophils $<0.5$ were excluded from this analysis with no change in distribution. SEM: standard error of the mean and SD: standard deviation of the mean.

Table 3. Mean ( \pm standard deviation) of the neutrophil count with respect to gender and age group distribution.

\begin{tabular}{|c|c|c|c|c|}
\hline Age group (years) & \multicolumn{2}{|c|}{$13-40$} & \multicolumn{2}{|c|}{$41-60$} \\
\hline Gender & M & F & $\mathrm{M}$ & $\mathrm{F}$ \\
\hline Jeddah & $3.18 \pm 1.36$ & $3.22 \pm 1.36$ & $3.12 \pm 1.32$ & $3.17 \pm 1.34$ \\
\hline$P$ values & \multicolumn{2}{|c|}{$0.34^{*}$} & \multicolumn{2}{|c|}{$0.09^{* *}$} \\
\hline Taif & $3.10 \pm 1.37$ & $3.20 \pm 1.35$ & $3.19 \pm 1.33$ & $3.05 \pm 1.36$ \\
\hline$P$ values & \multicolumn{2}{|c|}{$0.73^{*}$} & \multicolumn{2}{|c|}{$0.41^{* *}$} \\
\hline Albaha & $2.741 \pm .29$ & $2.77 \pm 1.27$ & $2.76 \pm 1.30$ & $2.69 \pm 1.32$ \\
\hline P values & \multicolumn{2}{|c|}{$0.1^{*}$} & \multicolumn{2}{|c|}{$0.13^{* *}$} \\
\hline Abha & $2.76 \pm 1.26$ & $2.74 \pm 1.34$ & $2.75 \pm 1.31$ & $2.81 \pm 1.35$ \\
\hline P values & \multicolumn{2}{|c|}{$0.8^{*}$} & \multicolumn{2}{|c|}{$0.91^{* *}$} \\
\hline Khamis & $2.90 \pm 1.35$ & $2.94 \pm 1.36$ & $2.85 \pm 1.29$ & $2.87 \pm 1.33$ \\
\hline P values & \multicolumn{2}{|c|}{$0.001^{*}$} & \multicolumn{2}{|c|}{$0.01^{* *}$} \\
\hline Najran & $2.52 \pm 1.19$ & $2.62 \pm 1.26$ & $2.48 \pm 1.14$ & $2.61 \pm 1.28$ \\
\hline$P$ values & \multicolumn{2}{|c|}{$0.71^{*}$} & \multicolumn{2}{|c|}{$0.34^{* *}$} \\
\hline Gizan & $2.39 \pm 1.17$ & $2.49 \pm 1.20$ & $2.36 \pm 1.11$ & $2.44 \pm 1.15$ \\
\hline$P$ values & \multicolumn{2}{|c|}{$0.001^{*}$} & \multicolumn{2}{|c|}{$0.01^{* *}$} \\
\hline
\end{tabular}


$5 \%$ trimmed mean reported. Independent Student's t-test was used

* Comparison for gender.

** Comparison for age group.

While some comparison is statistically significant, the difference between the mean neutrophil counts is not clinically significant.

\section{Discussion}

The present study was conducted to authenticate our previous findings of a high prevalence of isolated neutropenia and explore any geographical association by including other cities and a large dataset of participants. After excluding abnormal tests results, 91,880 CBCs were included for determining the frequency of neutropenia in the study population. The current study validates our previous findings in which the prevalence of neutropenia was found to be $18 \%$. The findings of this study are consistent with those of Gari et al. [10] who also reported a high prevalence (20\%) of neutropenia in Jeddah. Several other studies have shown a variable frequency of neutropenia among different populations [9,13-15]. Moreover, the findings of this study indicated that the $2.5^{\text {th }}$ percentile of neutrophil count was lower than what is accepted now as lower end of the reference range; $1.5 \times 10^{9} / \mathrm{L}$, in all seven cities. This finding is most likely explained by racial effect. We postulate, as explained below, that this prevalence is most likely explained by Benign ethnic neutropenia.

BEN has a positive association with Duffy-null phenotype [16]. Atypical chemokine receptor-1 (ACKR1) gene, also known as the Duffy antigen receptor for chemokines (DARC), encodes the Duffy antigens $\left(\mathrm{Fy}^{\mathrm{a}}\right.$ and $\left.\mathrm{Fy}^{\mathrm{b}}\right)$. A single nucleotide polymorphism (SNP) at codon 42 (G125A, rs12075) results in two types of encoded proteins, Fya and Fy'. Fya antigen includes glycine, while Fy ${ }^{b}$ contains aspartic acid [17]. The Duffy-null phenotype $\left(\mathrm{Fy}^{\mathrm{a}-\mathrm{b}-}\right)$, also symbolized as erythroid silent (ES), occurs due to another SNP, rs2814778 at position $46(-46 \mathrm{~T}>\mathrm{C})$. The Duffy antigen expression is inhibited in individuals with homozygous C/C polymorphisms [18]. Individuals with rs2814778 suffer from an abnormal phenotype of neutrophils with enhanced egression into the tissues from the blood [19]. Plasmodium vivax enters the red cells through DARC. Duffy-null phenotypes therefore may provide a natural resistance to $P$. vivax. Consequently, this protective role of Duffy-null phenotypes in malaria endemic regions has undergone positive selection leading to its high prevalence in some ethnicities [20]. Although molecular analysis for rs2814778 of Duffy-null phenotypes was not conducted in this study to corelate the high frequency of this phenotype with BEN, the prevalence of Duffy-null phenotypes in Saudi Arabia reported in various studies could be linked with BEN. Duffy-null phenotype (Fyab-) at a frequency of 62 to $78 \%$ have been reported in Saudi Arabia populations [21,22]. Therefore, the isolated neutropenia reported in this current study may be Duffy-null phenotype-induced BEN.

We previously noticed a higher prevalence of isolated neutropenia at a high altitude in comparison with sea level [12]. In this study, the prevalence of isolated neutropenia in Taif city $(1,898 \mathrm{~m}$ above sea level) was not different from that in the coastal Jeddah city in the current study. Furthermore, in this study, we did not find any association between altitude levels and plasma volumes. Nonetheless, owing to many confounding factors, including different prevalence of BEN, the effect of altitude on the neutrophil count should not be dismissed without further research.

The ANC, usually above $1.5 \times 10^{9} / \mathrm{L}$, is used as an inclusion criterion for clinical trials [23]. This can potentially lead to exclusion of otherwise healthy individuals with physiologically lower neutrophil counts [24]. The same criterion is also used to grade chemotherapy-associated toxicity, which helps in dose adjustment of crucial treatments. Dose intensity of chemotherapy is important, and dose reduction or delay is associated with increased mortality [25,26]. In high-prevalence BEN populations, low ANC should not be considered a factor for altering the treatment strategies in certain clinical conditions. For instance, use of clozapine and deferiprone also induces neutropenia. Administration of 
these drugs can commence if the ANC is above $0.5 \times 10^{9} / \mathrm{L}$, in the absence of any other comorbid condition. In patients with malignancies, if the ANC ranges between $0.5 \times 10^{9} / \mathrm{L}$ and $1.0 \times 10^{9} / \mathrm{L}$, chemotherapy may be administered safely [27].

In clinical practice, individuals with ANC $<1.5 \times 10^{9} / \mathrm{L}$ usually undergo unwarranted laboratory and clinical investigations without any significant findings. Although the differential diagnoses of neutropenia are wide, BEN can be determined based on the appropriate clinical scenario. BEN is usually an incidental finding of isolated mild neutropenia with no other hematological abnormalities. It is not associated with increased risks of infections [28]. Previous studies have indicated the chronicity of the condition, and when absent, follow-up usually indicates the stability and benign nature of the condition [29]. Clinical history can also suggest the presence of other causes of neutropenia. For instance, in cyclic neutropenia, recurrent infections, especially oral lesions and low neutrophil counts, at 21-day intervals are common. Meanwhile, in BEN, recurrent infections are infrequent, while ANC remains low. Moreover, since molecular analysis for rs 2814778 of Duffy-null phenotypes is not available in clinical laboratories, the Duffy phenotype could be utilized as a marker for identifying individuals with BEN. In current clinical practice, the diagnosis of BEN depends on the exclusion of other causes of neutropenia.

This study has the advantage of large sample size and the availability of extended lab tests to exclude many health conditions. Nonetheless, it has some limitations. It was a retrospective analysis with few demographics and health-related data. Some micronutrient deficiencies that may affect neutrophil counts such as B12 and folate deficiencies were not assessed.

\section{Conclusions}

Isolated neutropenia is common in the southern and southwestern Saudi Arabia. BEN, extensively reported in Middle Eastern countries, is likely to explain many cases of mild isolated neutropenia. However, the effects of other factors, including geography, altitude, and autoimmune diseases, should be explored. In light of the above results, we suggest that the $2.5^{\text {th }}$ percentile of ANC for Saudi Arabians could be set at $1.0 \times 10^{9} / \mathrm{L}$. Additionally, ANC of $>0.8 \times 10^{9} / \mathrm{L}$, when isolated, chronic and stable with benign medical history, should not be considered alarming in the absence of fever, pyogenic infection, or inflammation.

Author Contributions: All authors contributed equally to the design, data collection, analysis and writing, and approval of the final manuscript. All authors have read and agreed to the published version of the manuscript.

\section{Funding: None}

Data Availability Statement: Data is available to readers upon reasonable request.

Conflicts of Interest: The authors declare no conflict of interest.

\section{References}

1. Nauseef, W.M. Neutrophils, from Cradle to Grave and Beyond. Immunological Reviews 2016, 273, 5-10, doi:https://doi.org/10.1111/imr.12463.

2. Kobayashi, Y. Neutrophil Biology: An Update. EXCLI J 2015, 14, 220-227, doi:10.17179/excli2015-102.

3. Hoffbrand AV, M.P. The white cells 1: granulocytes, monocytes and their benign disorders. In Hoffbrand's Essential Hematology; Hoffbrand AV, M.P., Ed.; Wiley-Blackwell, 2016; pp. 87-101.

4. Peterson, R.F.; Peterson, W.G. The Differential Count at High Altitudes. The Journal of Laboratory and Clinical Medicine 1935, 20, 723-726, doi:10.5555/uri:pii:S0022214335909003.

5. Atallah-Yunes, S.A.; Ready, A.; Newburger, P.E. Benign Ethnic Neutropenia. Blood Reviews 2019, 37, 100586, doi:10.1016/j.blre.2019.06.003. 
6. Lakhotia, R.; Aggarwal, A.; Link, M.E.; Rodgers, G.P.; Hsieh, M.M. Natural History of Benign Ethnic Neutropenia in Individuals of African Ancestry. Blood Cells, Molecules, and Diseases 2019, 77, 12-16, doi:10.1016/j.bcmd.2019.01.009.

7. Phillips, D.; Rezvani, K.; Bain, B.J. Exercise Induced Mobilisation of the Marginated Granulocyte Pool in the Investigation of Ethnic Neutropenia. Journal of Clinical Pathology 2000, 53, 448-483, doi:10.1136/jcp.53.6.481.

8. Souto Filho, J.T.D.; Portugal, R.D.; Nucci, M. Effect of Circadian Variation on Neutrophil Mobilization to the Peripheral Blood in Benign Constitutional Neutropenia. Experimental Hematology 2019, 69, 22-26, doi:10.1016/j.exphem.2018.10.007.

9. Denic, S.; Narchi, H.; Al Mekaini, L.A.; Al-Hammadi, S.; Al Jabri, O.N.; Souid, A.K. Prevalence of Neutropenia in Children by Nationality. BMC Hematology 2016, 16, 1-7, doi:10.1186/s12878-016-0054-8.

10. Gari, M.; Dakhakhni, M.; Gari, A.; Alshihri, E.; Al-Jahdali, R.; Narasimhan, K.; Liang, S.; Al-Sayes, F.; Kalamegam, G.; Chaudhary, A.; et al. Incidence and Potential Causative Factors Associated with Chronic Benign Neutropenia in the Kingdom of Saudi Arabia. BMC Proceedings 2015, 9, 1-7, doi:10.1186/1753-6561-9-S2-S1.

11. Ortiz, M. V.; Meier, E.R.; Hsieh, M.M. Identification and Clinical Characterization of Children with Benign Ethnic Neutropenia. Journal of Pediatric Hematology/Oncology 2016, 38, e140-e143, doi:10.1097/MPH.0000000000000528.

12. Alkhaldy, H.Y.; Awan, Z.A.; Abouzaid, A.A.; Elbahaie, H.M.; Amoudi, S.M.A.; Andarawi, M.; Shehata, S.F. The Prevalence of Isolated Neutropenia at High Altitude in Southern Saudi Arabia: Does Altitude Affect Leucocyte Count? 2020, 2020:13, 1373-1379, doi:doi.org/10.2147/IJGM.S284133.

13. Wonodi, I.; Oduguwa, T.; Amoo, I.; Adebayo, A.; Adebimpe, T.; Kalejaiye, O.; Adebayo, R.; Lawal, R.; Peters, O.; Olaniyan, A.; et al. 120. Benign Ethnic Neutropenia in a Sample of Nigerian Healthy Controls and ClozapineTreated Schizophrenia Patients With DARC Null Variant. Schizophrenia Bulletin 2017, 43, S67-S67, doi:10.1093/schbul/sbx021.178.

14. Goswami, R.; Kakkar, N.; John, M.J. Lower Total Leukocyte and Neutrophil Counts in Healthy Young Africans from Uganda. Indian J Hematol Blood Transfus 2018, 34, 278-281, doi:10.1007/s12288-017-0866-0.

15. Merz, L.E.; Achebe, M. When Non-Whiteness Becomes a Condition. Blood 2020, doi:10.1182/blood.2020008600.

16. Rappoport, N.; Simon, A.J.; Lev, A.; Yacobi, M.; Kaplinsky, C.; Weingarten, M.; Somech, R.; Amariglio, N.; Rechavi, G. Correlation between "ACKR1/DARC Null” Polymorphism and Benign Neutropenia in Yemenite Jews. Br J Haematol 2015, 170, 892-895, doi:10.1111/bjh.13345.

17. Rappoport, N.; Simon, A.J.; Amariglio, N.; Rechavi, G. The Duffy Antigen Receptor for Chemokines, ACKR1,'Jeanne DARC' of Benign Neutropenia. British Journal of Haematology 2019, 184, 497-507, doi:10.1111/bjh.15730.

18. Fragiadaki, I.; Papadakis, S.; Sevastaki, G.; Sfyridaki, K.; Mavroudi, I.; Goulielmos, G.N.; Kanellou, P.; Mörtberg, A.; Höglund, P.; Gemenetzi, K.; et al. Increased Frequency of the Single Nucleotide Polymorphism of the DARC/ACKR1 Gene Associated with Ethnic Neutropenia in a Cohort of European Patients with Chronic Idiopathic Neutropenia. American Journal of Hematology 2020, 95, E163-E166, doi:10.1002/ajh.25813.

19. Palmblad, J.; Höglund, P. Ethnic Benign Neutropenia: A Phenomenon Finds an Explanation. Pediatric Blood E Cancer 2018, 65, e27361, doi:10.1002/pbc.27361.

20. Dinardo, C.L.; Kerbauy, M.N.; Santos, T.C.; Lima, W.M.; Dezan, M.R.; Oliveira, V.B.; Mendrone-Júnior, A.; Rocha, V.; Velloso, E.D.R.P. Duffy Null Genotype or Fy(a-b-) Phenotype Are More Accurate than Self-Declared Race for Diagnosing Benign Ethnic Neutropenia in Brazilian Population. International Journal of Laboratory Hematology 2017, 39, e144-e146.

21. Fahad Almsned, L.A. Frequency of the ABO and Duffy Phenotypes among Saudi Population in Eastern Provence. Journal of Medical Science And clinical Research 2017, 05, 19295-19298, doi:10.18535/jmscr/v5i3.154. 
22. Owaidah, A.Y.; Naffaa, N.M.; Alumran, A.; Alzahrani, F. Phenotype Frequencies of Major Blood Group Systems (Rh, Kell, Kidd, Duffy, Mns, p, Lewis, and Lutheran) among Blood Donors in the Eastern Region of Saudi Arabia. Journal of Blood Medicine 2020, 11, 59-65, doi:10.2147/JBM.S236834.

23. Jin, S.; Pazdur, R.; Sridhara, R. Re-Evaluating Eligibility Criteria for Oncology Clinical Trials: Analysis of Investigational New Drug Applications in 2015. Journal of Clinical Oncology 2017, 35, 3745-3752, doi:10.1200/JCO.2017.73.4186.

24. Vastola, M.E.; Yang, D.D.; Muralidhar, V.; Mahal, B.A.; Lathan, C.S.; McGregor, B.A.; Nguyen, P.L. Laboratory Eligibility Criteria as Potential Barriers to Participation by Black Men in Prostate Cancer Clinical Trials. JAMA Oncol 2018, 4, 413-414, doi:10.1001/jamaoncol.2017.4658.

25. Denduluri, N.; Lyman, G.H.; Wang, Y.; Morrow, P.K.; Barron, R.; Patt, D.; Bhowmik, D.; Li, X.; Bhor, M.; Fox, P.; et al. Chemotherapy Dose Intensity and Overall Survival Among Patients With Advanced Breast or Ovarian Cancer. Clinical Breast Cancer 2018, 18, 380-386, doi:10.1016/j.clbc.2018.02.003.

26. Crawford, J.; Denduluri, N.; Patt, D.; Jiao, X.; Morrow, P.K.; Garcia, J.; Barron, R.; Lyman, G.H. Relative Dose Intensity of First-Line Chemotherapy and Overall Survival in Patients with Advanced Non-Small-Cell Lung Cancer. Supportive Care in Cancer 2020, 28, 925-932, doi:10.1007/s00520-019-04875-1.

27. Hsieh, M.M.; Tisdale, J.F.; Rodgers, G.P.; Young, N.S.; Trimble, E.L.; Little, R.F. Neutrophil Count in African Americans: Lowering the Target Cutoff to Initiate or Resume Chemotherapy? Journal of Clinical Oncology 2010, 28, 1633-1637.

28. Legge, S.E.; Christensen, R.H.; Petersen, L.; Pardiñas, A.F.; Bracher-Smith, M.; Knapper, S.; Bybjerg-Grauholm, J.; Baekvad-Hansen, M.; Hougaard, D.M.; Werge, T.; et al. The Duffy-Null Genotype and Risk of Infection. Human Molecular Genetics 2020, 00, 1-9, doi:10.1093/hmg/ddaa208.

29. Donadieu, J.; Beaupain, B.; Fenneteau, O.; Bellanné-Chantelot, C. Congenital Neutropenia in the Era of Genomics: Classification, Diagnosis, and Natural History. British Journal of Haematology 2017, 179, 557-574. 